\title{
WW domain-containing oxidoreductase in neuronal injury and neurological diseases
}

\author{
Hsin-Tzu Chang ${ }^{1}$, Chan-Chuan Liu ${ }^{1}$, Shur-Tzu Chen ${ }^{1}$, Ye Vone Yap ${ }^{2}$, Nan-Shang \\ Chang ${ }^{2,3}$ and Chun-I Sze ${ }^{1}$ \\ ${ }^{1}$ Department of Cell Biology and Anatomy, College of Medicine, National Cheng Kung University, Tainan, Taiwan \\ 2 Institute of Molecular Medicine, College of Medicine, National Cheng Kung University, Tainan, Taiwan \\ ${ }^{3}$ Advanced Optoelectronic Technology Center, National Cheng Kung University, Tainan, Taiwan \\ Correspondence to: Nan-Shan Chang, email: changns@mail.ncku.edu.tw
}

Chun-l Sze, email: szec@mail.ncku.edu.tw

Keywords: WWOX, WOX1, neurodegeneration, neurites, neuronal death, transcription factors

Received: November 14,2014 Accepted: December 09, $2014 \quad$ Published: December 10, 2014

This is an open-access article distributed under the terms of the Creative Commons Attribution License, which permits unrestricted use, distribution, and reproduction in any medium, provided the original author and source are credited.

\section{ABSTRACT}

The human and mouse WWOX/Wwox gene encodes a candidate tumor suppressor WW domain-containing oxidoreductase protein. This gene is located on a common fragile site FRA16D. WWOX participates in a variety of cellular events and acts as a transducer in the many signal pathways, including TNF, chemotherapeutic drugs, UV irradiation, Wnt, TGF- $\beta$, C1q, Hyal-2, sex steroid hormones, and others. While transiently overexpressed WWOX restricts relocation of transcription factors to the nucleus for suppressing cancer survival, physiological relevance of this regard in vivo has not been confirmed. Unlike many tumor suppressor genes, mutation of WWOX is rare, raising a question whether $W W O X$ is a driver for cancer initiation. WWOX/ Wwox was initially shown to play a crucial role in neural development and in the pathogenesis of Alzheimer's disease and neuronal injury. Later on, WWOX/Wwox was shown to participate in the development of epilepsy, mental retardation, and brain developmental defects in mice, rats and humans. Up to date, most of the research and review articles have focused on the involvement of WWOX in cancer. Here, we review the role of WWOX in neural injury and neurological diseases, and provide perspectives for the WWOX-regulated neurodegeneration.

\section{INTRODUCTION}

Human and mouse WWOX/Wwox gene was first cloned in year 2000 [1-5; reviews]. Later on, the mouse Wwox genome, which has one million bases, was isolated [6]. WW domain-containing oxidoreductase (designated WWOX, FOR, or WOX1) is a candidate tumor suppressor. This 46-KDa protein has two $N$-terminal WW domains and one $C$-terminal SDR (short-chain alcohol dehydrogenase/ reductase), plus a nuclear localization signal located in between the WW domains. Human WWOX gene, encoding the WWOX protein, has been mapped to a fragile site on the chromosome ch16q23.3-24.1 [1-5; reviews]. WW domains have been shown to interact with a wide variety of signaling proteins and functioning as adaptor proteins, transcriptional co-activators, and probably ubiquitin ligases. The first WW domain of WWOX binds a broad spectrum of PPxY-containing proteins, including p63, p73, AP-2 $\gamma$ (Activator protein $2 \gamma)$, ErbB4 (v-Erb-B2 avian erythroblastic leukemia viral oncogene homolog 4), Runx2 (Runt-related transcription factor 2), Dvl-2 (Dishevelled homolog protein-2), SIMPLE (Small integral membrane protein of the lysosome/late endosome), MET (MET proto-oncogene), LMP2A (viral latent membrane protein $2 \mathrm{~A}$ ), and others [1-13]. In stark contrast, when WWOX becomes Tyr33-phosphorylated in the first WW domain, it acquires an enhanced binding capability with PPxY motifdeficient proteins such as p53, JNK1 (c-Jun $N$-terminal kinase 1), c-Jun (Jun proto-oncogene), CREB (cAMP responsive element binding protein), and Zfra (Zinc finger-like protein that regulates apoptosis) [4,8,10-14].

Additionally, the $C$-terminal SDR domain of WWOX physically interacts with membrane hyaluronidase Hyal-2 [15], tau [16] and GSK-3 $\beta$ (Glycogen synthase 
kinase 3 beta) [17]. We have shown that WWOX is an inhibitor of neurodegeneration, because of its interaction with tau and inhibition of enzyme-dependent Tau hyperphosphorylation $[16,17]$. WWOX is involved in the TGF- $\beta$ (Transforming growth factor beta) signaling [15]. Hyal-2 binds TGF- $\beta 1$ and acts as a receptor. During signaling, Hyal-2 is internalized and recruits WWOX via binding to the SDR domain, and the resulting WWOX/ Hyal-2 complex binds Smad4 and is then accumulated in the nucleus, which affects cell survival or death [15]. WWOX physically interacts with MEK1 (MitogenActivated Protein Kinase Kinase 1), and that dissociation of the protein complex results in apoptosis of leukemia cells [18]. Förster resonance energy transfer (FRET) analysis revealed that both the $N$-terminal WW domain and the $C$-terminal SDR domain are capable of interacting with MEK1 [18].

Supporting evidence from Drosophila and mouse knockout models has revealed that WWOX acts more than just a tumor suppressor [7-12]. Overexpression of the full-length WWOX or its WW or SDR domain region induces apoptosis $[4,8,19]$. WWOX enhances tumor necrosis factor (TNF) cytotoxicity by down-regulation of the apoptosis inhibitors Bcl-2 and Bcl-xL. Under stress conditions, activated or Tyr33-phsphorylated WWOX binds $\mathrm{p} 53$, in which the complex co-translocates to the mitochondria or to the nucleus $[4,8,20]$. WWOX in the cellular or nuclear compartment may interfere with genes transcription or cancers response to chemotherapy [13,21].

WWOX is involved in binding and regulating GSK$3 \beta$ activity, and this limits Tau hyperphosphorylation, neurite outgrowth in neuronal differentiation, and formation of neurofibrillary tangles (NFTs) and senile plaques in Alzheimer's disease (AD) [16,17,22]. Neural injuries to the brain, spinal cord, or peripheral nerve are devastating, which often leads to complex and lifelong disability. These injuries could be acute or chronic and continuously affect the remaining undamaged nervous system. Neural injuries cause damage to the neurons, its processes or neurites, and neural supporting cell or glial cells. Data collect from Genome Wide Association Studies (GWAS) and full knock-out (KO) mice models have implicated that $W W O X$ gene may be associated with metabolic syndrome and related conditions that affecting cardiovascular and neurological systems [23-27]. Given WWOX interacts with molecules involved in cell signaling, gene transcription, and lipid metabolism, all of which may regulate cell survival or death. It is very likely that WWOX plays a critical role in central nervous system (CNS) physiology and injury. In this article, we review the possible perspectives that WWOX may be involved in neural injury and its potential role in the pathogenesis of neurological diseases.

\section{WWOX in neuronal death signaling}

Cell death occurs in neural injury or neurodegenerative diseases. The level of TNF receptor I (TNFR1) is up-regulated in $\mathrm{AD}$, which correlates with the apoptotic process through its ligand TNF- $\alpha$ (tumor necrosis factor alpha) [28-30]. TNF- $\alpha$ induces inflammatory response and apoptosis by activating TRADD (TNF receptor-associated protein with death domain), FADD (Fas-associated protein with death domain), JNK1, WWOX, and NF- $\mathrm{BB}$ (nuclear factor-kappa B) [1,2,2830]. TNF- $\alpha$ induces activation of JNK1 in AD patients and mouse models of $\mathrm{AD}$, as evidenced by the expression of pro-apoptotic genes and activation of caspases- 3 and caspase-9 [28-30]. Additionally, sciatic nerve transection could lead to neuronal injury and death. This effect rapidly results in activation of JNK1 and WWOX as short as 30 min in the injured DRG neurons in rats. Subsequently, there are significantly increased accumulation of WWOX, JNK1, CREB, c-Jun, NF- $\mathrm{kB}$ and ATF3 in the nuclei of injured large neurons within 24 hours or during the first week of the injury [31] (Figure 1). Later, in the chronic phase of the neuronal injury, concurrent activation of WWOX, CREB, and NF- $\mathrm{kB}$ occurs in small neurons prior to apoptosis [31]. WWOX strongly binds CREB in the nuclei. Additionally, WWOX blocks the promoter activation governed by the prosurvival CREB, CRE and $\mathrm{AP}-1$ in vitro. In contrast, WWOX enhances the promoter activation regulated by c-Jun, Elk-1 and NF- $\mathrm{\kappa B}$ [31]. Whether WWOX regulates the function of transcription factors in neuronal survival or death in vivo remains to be established.

WWOX interacts with p53 and both proteins act synergistically to induce apoptosis $[4,8,10,14,19]$. Intriguingly, soluble $\mathrm{A} \beta$ (amyloid beta) peptides are involved in HIPK2 (Homeodomain-interacting protein kinase 2) degradation, which results in misfolded p53 and altered vulnerability of cells to noxious stimulus, suggesting that conformational changed p53 can be a putative biomarker for $\mathrm{AD}[32,33]$. Whether or not WWOX interacts with $A \beta$ peptides or HIPK2 remains to be established; however, it is important to discern the link between transient overexpression of WWOX or SDR domain and accumulation of $A \beta$ peptide, Tau phosphorylation, and formation of NFTs.

\section{WWOX in neurological disease pathology}

Tau is a microtubule-associated protein functioning to promote microtubule assembly and is essential for the axonal transportation. Phosphorylation of Tau affects axonal flow and cell viability during differentiation [32]. Tau phosphorylation can be regulated by WWOX via its interaction with GSK-3 $\beta$, JNK1, ERK, Wnt/betacatenin and Tau $[14,16-18,33-36]$. GSK-3 $\beta$ maintains a 
hyperactive state and hyperphosphorylates Tau in AD. GSK-3 $\beta$ regulates APP (amyloid precursor protein) metabolism and overproduction of $\mathrm{A} \beta$ that leads to reduced neurogenesis and increased apoptosis [37]. Protein phosphatase 2A (PP2A) is shown to be a key enzyme in dephosphorylating Tau [38].

WWOX binds directly to Tau through its SDR domain. Silencing of WWOX by small interfering RNA increases the binding of Tau to GSK-3 $\beta$ and Tau phosphorylation, indicating that WWOX is involved in regulating GSK-3 $\beta$ activity [16]. Overexpression of WWOX enhances the SH-SY5Y cell differentiation with or without the treatment of retinoic acid (RA). In contrast, knockdown of WWOX in RA-differentiated SH-SY5Y cells causes a decrease in neurite outgrowth, suggesting a role of WWOX in neuronal differentiation [17]. The physical interactions of WWOX with Tau, JNK1 and GSK-3 $\beta$ have been demonstrated in the rat brains extract and cultured cells $[16,17]$. These findings suggest that
WWOX may participate in AD pathology through its protein-binding partners.

WW domain-containing proteins participate in the TGF- $\beta$ signaling [15]. TGF- $\beta 1$ is crucial in regulating neuroprotection and neurodegeneration [39-41] (Figure $2)$. Recently, we have identified that a small TGF- $\beta 1$ induced antiapoptotic factor (TIAF1) is involved in the pathogenesis of AD [40]. Long-term TGF- $\beta 1$ exposure results in irreversible formation of amyloid fibrils and apolipoprotein $\mathrm{E}$ (ApoE) depositions in vivo, even after silencing of the transgene or under TGF- $\beta 1$ removal [39]. Both WWOX and TIAF1 participate in regulating the activation of Smad-driven promoter via type II TGF- $\beta 1$ receptor (T $\beta$ RII)-independent manner to induce apoptosis or neurodegeneration $[15,40]$. The differences of TGF- $\beta 1$ in exerting neuroprotection or degeneration may be related to the TIAF1/Smad4 complex formation, as Smad4 limits the polymerization of TIAF1 [40]. WWOX is able to interact with TIAF1 (Chang et al., unpublished), whereas
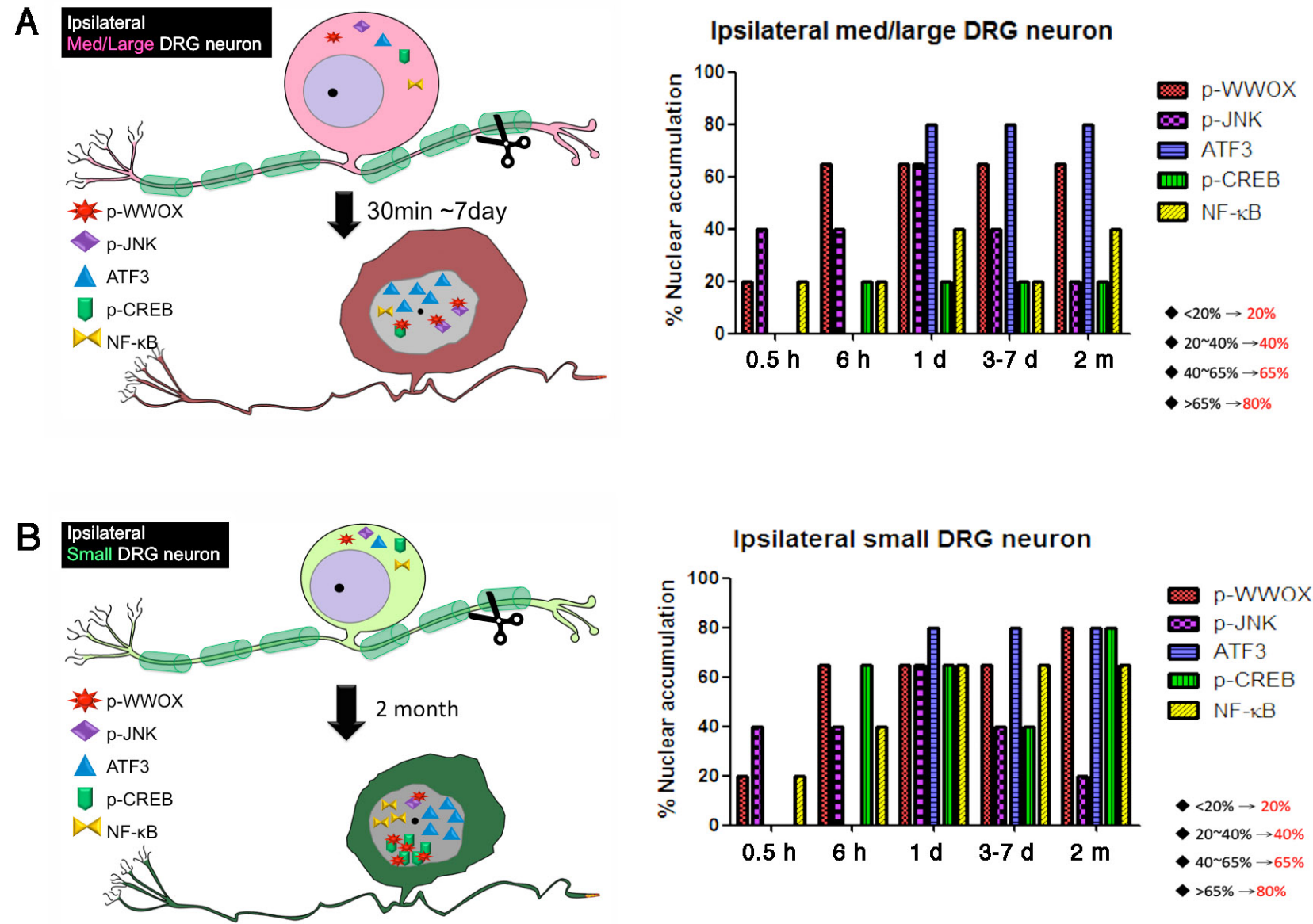

Figure 1: Relocation of WWOX and transcription factors to the nuclei upon neuronal injury. (A) Rats were subjected to sciatic nerve axotomy. During the acute phase of injury, activated p-JNK and p-WWOX rapidly relocated to the nuclei of injured neurons in $30 \mathrm{~min}$ in the ipsilateral side, followed by continual accumulation of activated transcription factors $\mathrm{CREB}, \mathrm{NF}-\mathrm{\kappa B}$, ATF3 and others to in the nuclei of injured medium/large-size neurons in 1 to 7 days. JNK and CREB are likely to block the proapoptotic function of WWOX via direct binding [31]. Neuronal death can occur in 7 days. (B) In the chronic phase, gradual accumulation of activated JNK, WWOX, CREB, ATF3 and NF-KB in the nuclei was found in the small neurons post injury for 2 months. The schematic graphs were drawn based on published data [31]. The percentages of protein nuclear accumulation are shown. The actual range of each bar is shown in the lower right. 
whether WWOX prevents TIAF1 aggregation is unknown. Aggregated TIAF1 induces apoptosis in a caspasedependent manner. Under physiological conditions, TGF- $\beta 1$ signals the binding of TIAF1 with Smad4 to form a complex, which relocates to the nuclei to modulate gene transcription [40]. Smad proteins are involved in transcribing the gene coding for membrane APP [41,42]. These studies further dissect the role of WWOX in TGF- $\beta 1$-induced TIAF1 self-aggregation and Smad4 overexpression in senile plaques formation, which might shed light for the development of therapeutic strategy in neurodegenerative diseases.

\section{WWOX in metabolic syndrome and neural development}

Complications from metabolic syndromes such as high blood cholesterol, hypertension, obesity, and diabetes interfere blood perfusion and energy supply to the nervous system and increase the risk of neuronal injury. Genome based gene analyses have identified $W W O X$ as a hypertension (HTN) susceptibility gene in Asian populations $[43,44]$. HTN candidate genes or HTN itself in humans and mice are associated with obesity, glucose metabolism, ion homeostasis, diabetic mellitus, and cardiovascular or neurological dysfunctions. All of which are important for the development of metabolic syndromes. In addition, $W W O X$ gene alteration is

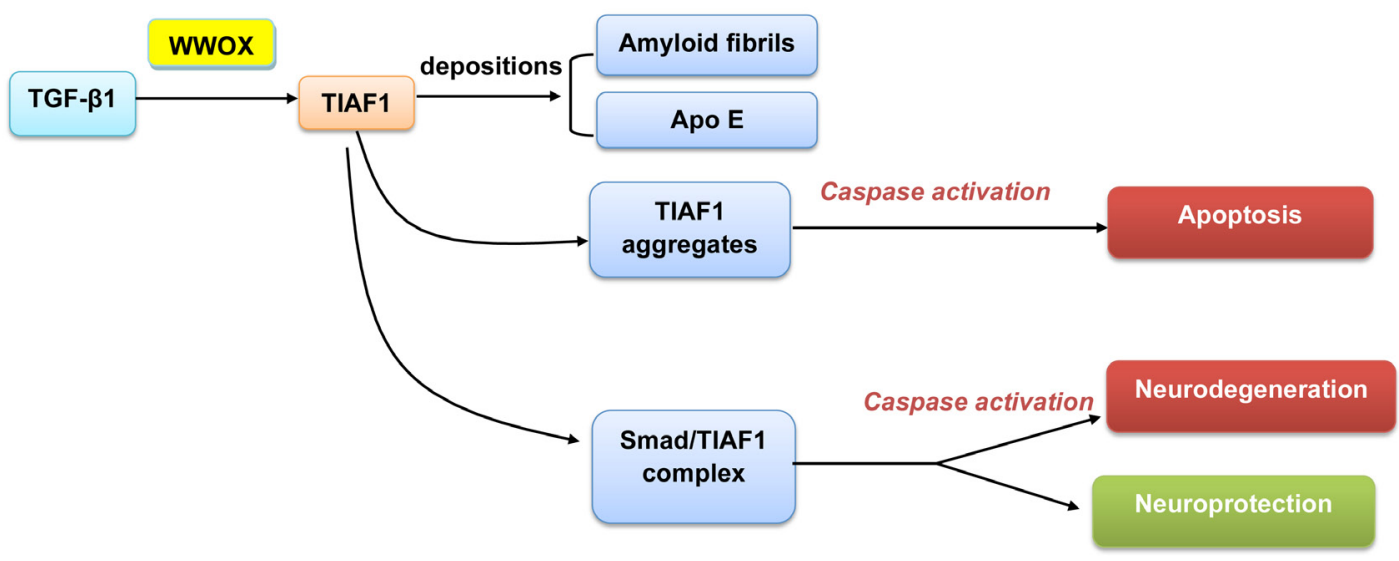

Figure 2: A schematic graph of TGF-ß1-regulated neurodegeneration. Both WWOX and TIAF1 participate in regulating apoptosis and neurodegeneration [15,40]. Aggregated TIAF1 induces apoptosis in a caspase-dependent manner, whereas Smad4 controls TIAF1 aggregation [40]. Long-term TGF- $\beta 1$ exposure may result in irreversible formation of amyloid fibrils and apolipoprotein E (ApoE) depositions in vivo [39].

\section{WWOX GENE}

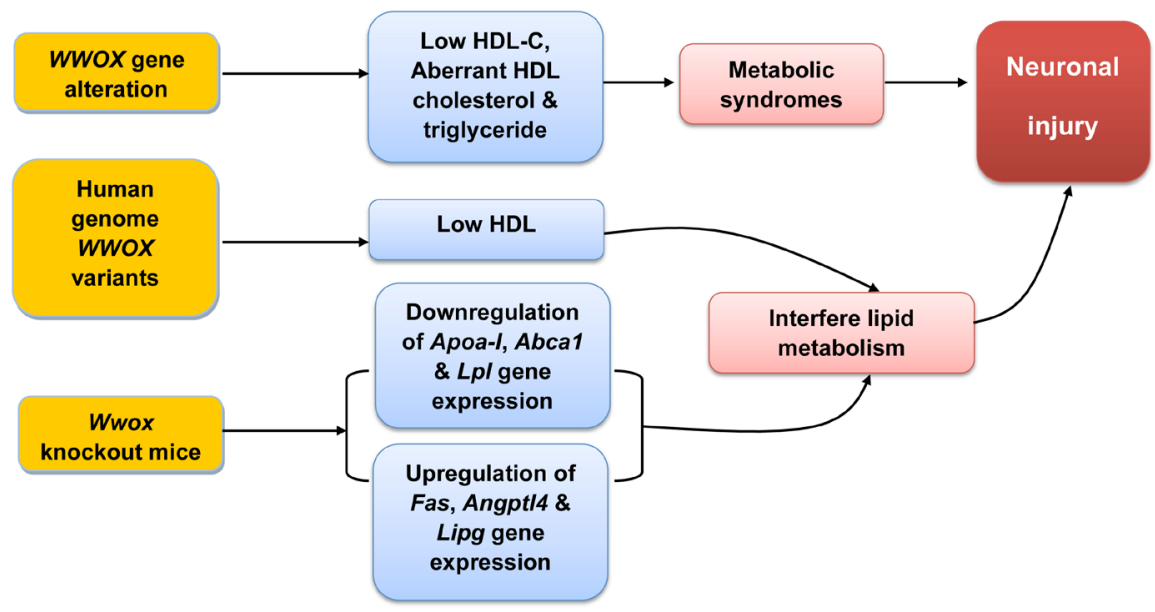

Figure 3: $\boldsymbol{W W O X}$ in metabolism and metabolic diseases. $W W O X$ gene alteration is associated with low plasma HDL-C levels and aberrant HDL cholesterol and triglyceride levels, and these may lead to the development of metabolic syndromes and chances of neuronal injury [45]. Eight variants have been identified in the human WWOX genome, which are significantly associated with the low-HDL trait [47]. In Wwox knockout mice, the expression levels of several genes and proteins are altered [47]. 
associated with low plasma HDL-C levels and aberrant HDL cholesterol and triglyceride levels, which are crucial for the development of metabolic syndromes and increases in the risk of neuronal injury [45] (Figure 3). Multiple metabolic defects occur in whole body and conditional Wwox knockout mice, further supporting Wwox gene as a key regulator in different metabolic processes $[45,46]$. Additionally, eight variants have been identified in the human WWOX genome, which are significantly associated with the low HDL trait in two multi-generational French Canadian dyslipidemic families [47] (Figure 3). Similarly, in whole body and liver conditional Wwox knockout mice, there are decreased protein levels of ApoA-I (apolipoprotein A-I) and ABCA1 (ATP-binding cassette transporter) levels in hepatic tissues, along with reduction in the mRNA expression of Apoa-I and Lpl (lipoprotein lipase), upregulation in Fas, Angptl4 (angiopoietin-like 4) and Lipg (endothelial lipase) [47] (Figure 3). These observations suggest a significant role for WWOX in modulating HDL and lipid metabolism, including cholesterol homeostasis, ApoA-I/ABCA1 pathway, and fatty acid biosynthesis/triglyceride metabolism [47]. Interference in lipid metabolism may be a critical contributor in the pathogenesis of neurological diseases.

Large consanguineous family studies have reported that homozygous $W W O X$ point mutations (e.g. P47T and G372R) could lead to a new form of childhood onset autosomal recessive cerebellar ataxia and epilepsy [26]. A recent case showed that homozygous nonsense mutation of $W W O X$ results in protein loss of function, and the patient suffers from severe anomalies, including short and growth retardation, microcephaly with seizure, retinal degeneration, and early death at 16 months of age [27]. In parallel, 13-bp deletion in exon 9 of $W$ wox gene has been found in Ide/Ide rat and this results in a frameshift reading of $W W O X$ codon at the $C$-terminus [48]. Nonetheless, WWOX protein is barely detectable in the testes and hippocampi of lde/lde rats, suggesting that the $C$-terminus of WWOX is critical to protein stability. In a striking similarity to those symptoms in humans, the lde/lde rats are shown to have dwarfism, postnatal lethality, male hypogonadism, and a high incidence of epilepsy and many vacuoles in the hippocampus and amygdala [48]. Nonetheless, despite the loss of function of WWOX protein, no tumor formation was shown in the aforementioned cases $[26,27,48]$. Overall, these reports clearly indicate that germline loss-of-function of $W W O X$ leads to developmental deficiency in the neural system.

In contrast to the aforementioned observations, functional deficiency of $W W O X$ in Drosophila fails to generate the disease phenotypes in the neural system [49]. It has been suggested that WWOX functions in aerobic glycolysis metabolism (Warburg effect) and regulates reactive oxygen species [49]. WWOX is functionally linked to either CG6439/isocitrate dehydrogenase (IDH) or $\mathrm{Cu}-\mathrm{Zn}$ superoxide dismutase $(S O D)$, whereas direct binding interactions among these proteins are unknown. These proteins may co-localize and function together in the mitochondria $[1,2,4]$. Oxidative phosphorylation increases a steady-state transcription of WWOX gene,
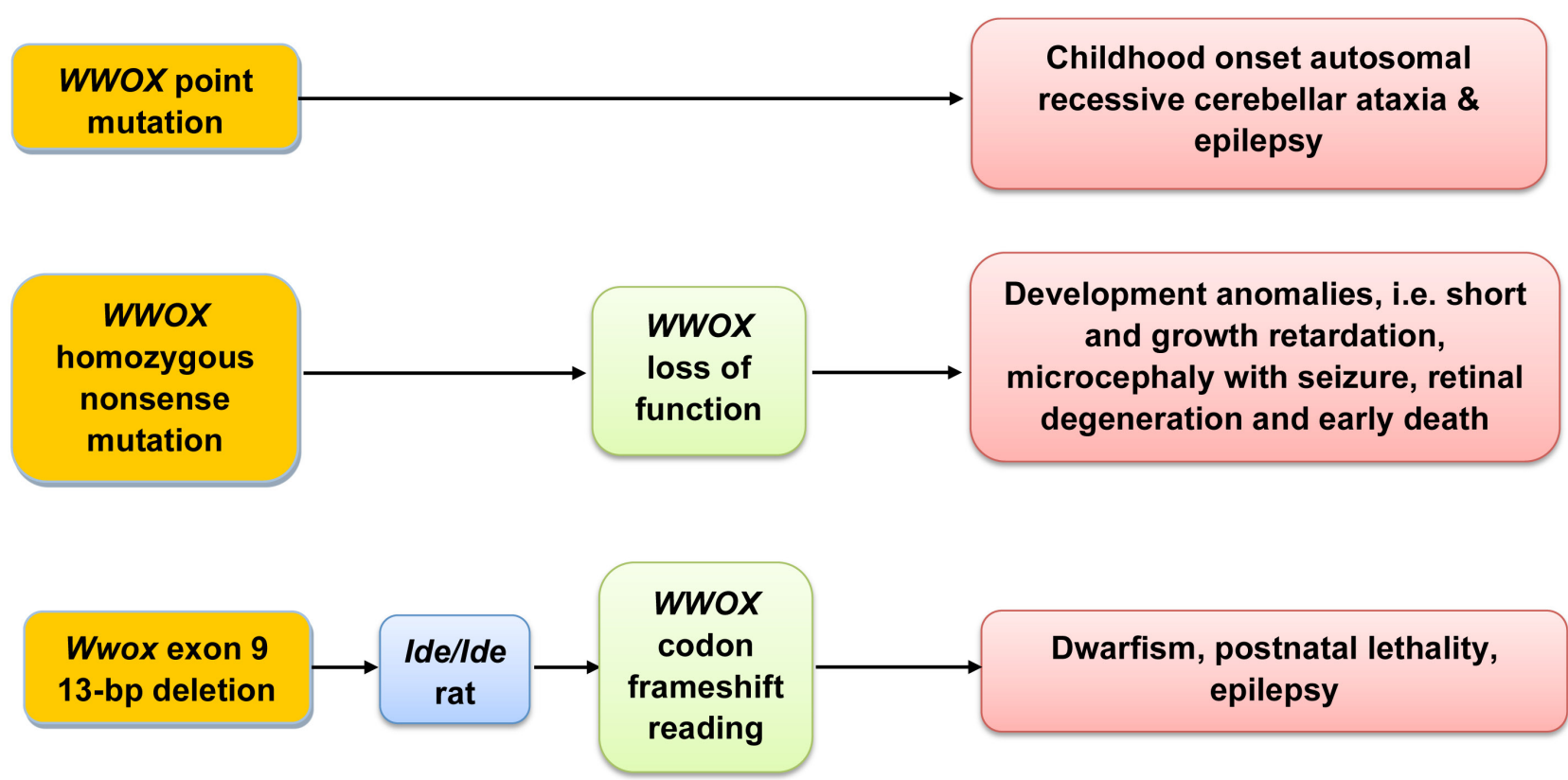

Figure 4: $\boldsymbol{W} \boldsymbol{W O X}$ gene mutations. Homozygous $W W O X$ point mutations (e.g. P47T and G372R) could lead to a new form of childhood onset autosomal recessive cerebellar ataxia and epilepsy [26]. Also, homozygous nonsense mutation of $W W O X$ causes protein loss of function, and the patient suffers from severe anomalies, including short and growth retardation, microcephaly with seizure, retinal degeneration, and early death at 16 months of age [27]. A 13-bp deletion in exon 9 of Wwox gene is found in Ide/Ide rat and this results in a frameshift reading of $W W O X$ codons at the $C$-terminus [48]. 
whereas glycolysis downregulates the expression [50]. The observation further supports the essential role of WWOX in the mitochondria, and that downregulation of WWOX in AD causes neuronal damage $[1,2,4]$.

\section{CONCLUSION AND PERSPECTIVES}

In conclusion, substantial evidence has shown that WWOX participates in the control of the function of transcription factors in vivo. For example, WWOX could significantly promote the NF- $\mathrm{KB}$-induced promoter activation [31]. WWOX also regulates the transcriptional activation of CREB, CRE, c-Jun, Elk-1 and AP-1 [31]. Apparently, under normal physiology, this regulatory control is likely to affect neuronal degeneration or regeneration and cell metabolism. $W W O X / W$ wox is involved in the maintenance of lipid metabolism [26,27,45-48]. Alterations of $W W O X / W w o x$ gene, including point mutation, missense or nonsense mutation, and deletion, may lead to ataxia, epilepsy, dementia, neurodegeneration, and diseases associated with HDL lipid metabolism [16,17,26,27,45-48]. Although overexpressed $W W O X / W$ wox may induce death of many types of cancer cells [1-11], no spontaneous cancer formation occurs in humans with nonsense mutation of this gene. Further, knockdown of WWOX/Wwox may readily induce apoptosis of many types of normal and cancer cells. Conceivably, WWOX plays a crucial role in cell survival by controlling metabolism. We have shown that the $C$-terminal SDR domain of WWOX is responsive to stimulation by sex steroid hormones androgen and estrogen, suggesting that WWOX may act as a hormone receptor [51,52]. To play this role, a portion of WWOX is located in the mitochondria to carry out its oxidoreductase function. Functional alteration of this event would cause the aforementioned metabolic diseases. Finally, p53 and WWOX are partners in inducing apoptosis [20]. In the absence of WWOX, p53 tends to become destabilized and subjected to degradation [20]. p53 is involved not only in tumor suppression but also in aging process and metabolic events [53,54]. Conceivably, the partnership between p53 and WWOX is crucial in the aging event and metabolic regulations.

\section{Author Contributions}

HTC, CCL and YVY: worked on graphic arts, literature search, and proofread manuscript; CIS, STC, and NSC: wrote the manuscript, conceived ideas, discussed thoroughly and proofread manuscript. HTC and YVY contributed equally to the preparation of this manuscript.

\section{Conflict of Interest Statement}

The authors declare that there is no conflict of interest in conducting the research.

\section{ACKNOWLEDGEMENTS}

This research was supported, in part, by the Department of Defense USA (W81XWH-08-1-0682), the Ministry of Science and Technology (MOST), Taiwan, ROC (NSC98-2628-B-006-041-MY3, 99-2320-B-006012-MY3, 102-2320-B-006-018-, 102-2320-B-006-030-, and 102-3011-P-006-005-), the National Health Research Institute, Taiwan, ROC (NHRI-EX101-10102BI, and NHRI-EX102-10102BI), the National Cheng Kung University Topnotch Projects (D103-24A01), and the Department of Health, Taiwan, ROC (DOH101-TD-PB111-TM010) (to NS Chang), and the MOST, Taiwan, ROC (NSC 96-2320- B-006-036-MY, 96-2320-B-006-053-, and 102-2320-B-006 -006-) to CI Sze.

\section{REFERENCES}

1. Chang NS. A potential role of p53 and WOX1 in mitochondrial apoptosis (review). Int J Mol Med. 2002; 9:19-24. Review.

2. Chang NS, Doherty J, Ensign A, Lewis J, Heath J, Schultz L, Chen ST, and Oppermann U. Molecular mechanisms underlying WOX1 activation during apoptotic and stress responses. Biochem Pharmacol. 2003; 66:1347-1354. Review.

3. Ludes-Meyers JH, Bednarek AK, Popescu NC, Bedford M, Aldaz CM. WWOX, the common chromosomal fragile site, FRA16D, cancer gene. Cytogenet Genome Res. 2003; 100:101-110.

4. Chang NS, Hsu LJ, Lin YS, Lai FJ, Sheu HM. WW domaincontaining oxidoreductase: a candidate tumor suppressor. Trends Mol Med. 2007; 13:12-22.

5. O'Keefe LV, Richards RI. Common chromosomal fragile sites and cancer: focus on FRA16D. Cancer Lett. 2006; 232:37-47. Review.

6. Krummel KA1, Denison SR, Calhoun E, Phillips LA, Smith DI. The common fragile site FRA16D and its associated gene WWOX are highly conserved in the mouse at Fra8E1. Genes Chromosomes Cancer. 2002; 34:154-167.

7. Del Mare S, Kurek KC, Stein GS, Lian JB, Aqeilan RI. Role of the WWOX tumor suppressor gene in bone homeostasis and the pathogenesis of osteosarcoma. Am J Cancer Res. 2011; 1:585-594.

8. Chang JY, He RY, Lin HP, Hsu LJ, Lai FJ, Hong Q, Chen SJ, Chang NS. Signaling from membrane receptors to tumor suppressor WW domain-containing oxidoreductase. Exp Biol Med (Maywood). 2010; 235:796-804. Review.

9. Salah Z, Aqeilan R, Huebner K. WWOX gene and gene 
product: tumor suppression through specific protein interactions. Future Oncol. 2010; 6:249-259. Review.

10. Chiang MF, Chou PY, Wang WJ, Sze CI, Chang NS. Tumor suppressor WWOX and p53 alterations and drug resistance in glioblastomas. Front Oncol. 2013; 3:43.

11. Chen SJ, Huang SS, Chang NS. Role of WWOX and NF$\kappa \mathrm{B}$ in lung cancer progression. Translational Respiratory Medicine. 2013; 1:15.

12. Gardenswartz A, Aqeilan RI. WW domain-containing oxidoreductase's role in myriad cancers: clinical significance and future implications. Exp Biol Med (Maywood). 2014; 239:253-263.

13. Salah Z, Bar-mag T, Kohn Y, Pichiorri F, Palumbo T, Melino G, Aqeilan RI. Tumor suppressor WWOX binds to $\triangle \mathrm{Np} 63 \alpha$ and sensitizes cancer cells to chemotherapy. Cell Death Dis. 2013; 4: e480.

14. Chang NS, Doherty J, Ensign A. JNK1 physically interacts with WW domain- containing oxidoreductase (WOX1) and inhibits WOX1-mediated apoptosis. J Biol Chem. 2003; 278:9195-9202

15. Hsu LJ, Schultz L, Hong Q, Van Moer K, Heath J, Li MY, Lai FJ, Lin SR, Lee MH, Lo CP, Lin YS, Chen ST, Chang NS. Transforming growth factor beta1 signaling via interaction with cell surface Hyal-2 and recruitment of WWOX/WOX1. J Biol Chem. 2009; 284:16049-16059.

16. Sze CI, Meng S, Pugazhenthi S, Jambal P, Hsu LJ, Heath J, Schultz L, Chang NS. Down-regulation of WW Domaincontaining oxidoreductase induces tau phosphorylation in vitro. J Biol Chem. 2004; 279:30498-30506.

17. Wang HY, Juo LI, Lin YT, Hsiao M, Lin JT, Tsai CH, Tzeng YH, Chuang YC, Chang NS, Yang CN, Lu PJ. WW domain-containing oxidoreductase promotes neuronal differentiation via negative regulation of glycogen synthase kinase 3ß. Cell Death Differ. 2012; 19:1049-1059.

18. Lin HP, Chang JY, Lin SR, Lee MH, Huang SS, Hsu LJ, Chang NS. Identification of an In Vivo MEK/WOX1 Complex as a Master Switch for Apoptosis in T Cell Leukemia. Genes Cancer. 2011; 2:550-562.

19. Chang NS, Pratt N, Heath J, Schultz L, Sleve D, Carey GB, Zevotek N. Hyaluronidase induction of a WW domaincontaining oxidoreductase that enhances tumor necrosis factor cytotoxicity. J Biol Chem. 2001; 276:3361-3370.

20. Chang NS, Doherty J, Ensign A, Schultz L, Hsu LJ, Hong Q. WOX1 is essential for tumor necrosis factor-, UV light-, staurosporine-, and p53-mediated cell death, and its tyrosine 33-phosphorylated form binds and stabilizes serine 46-phosphorylated p53. J Biol Chem. 2005; 280:4310043108.

21. Chiang MF, Yeh ST, Liao HF, Chang NS, Chen YJ. Overexpression of WW domain-containing oxidoreductase WOX1 preferentially induces apoptosis in human glioblastoma cells harboring mutant p53. Biomed Pharmacother. 2012; 66:433-438.

22. Hurtado DE, Molina-Porcel L, Carroll JC, MacDonald C,
Aboagye AK, Trojanowski JQ, Lee VM.Y. Selectively silencing GSK-3 isoforms reduces plaques and tangles in mouse models of Alzheimer's disease. J Neurosci. 2012; 32:7392-7402.

23. Willer CJ, Sanna S, Jackson AU, Scuteri A, Bonnycastle LL, Clarke R, et al. Newly identified loci that influence lipid concentrations and risk of coronary artery disease. Nat Genet. 2008; 40:161-169.

24. Saez ME, Gonzalez-Perez A, Martinez-Larrad MT, Gayan J, Real LM, Serrano-Rios M, Ruiz A. WWOX gene is associated with HDL cholesterol and triglyceride levels. BMC Med Genet. 2010; 11:148.

25. Vasan RS, Glazer NL, Felix JF, Lieb W, Wild PS, Felix SB, et al. Genetic variants associated with cardiac structure and function: a meta-analysis and replication of genome-wide association data. JAMA. 2009; 302:168-178.

26. Mallaret M, Synofzik M, Lee J, Sagum CA, Mahajnah M, Sharkia R, Drouot N, Renaud M, Klein FA, Anheim M, Tranchant C, Mignot C, Mandel JL, Bedford M, Bauer P, Salih MA, Schüle R, Schöls L, Aldaz CM, Koenig M. The tumour suppressor gene WWOX is mutated in autosomal recessive cerebellar ataxia with epilepsy and mental retardation. Brain. 2014; 137:411-419.

27. Abdel-Salam G, Thoenes M, Afifi HH, Korber F, Swan D, Bolz HJ. The supposed tumor suppressor gene WWOX is mutated in an early lethal microcephaly syndrome with epilepsy, growth retardation and retinal degeneration. Orphanet J Rare Dis. 2014; 9:12.

28. Agerman K, Baudet C, Fundin B, Willson C, Ernfors P. Attenuation of a caspase-3 dependent cell death in NT4and p75-deficient embryonic sensory neurons. Mol Cell Neurosci. 2000; 16:258-268.

29. Lee EJ, Han JE, Woo MS, Shin JA, Park EM, Kang JL, Moon PG, Baek MC, Son WS, Ko YT, Choi JW, Kim HS. Matrix metalloproteinase- 8 plays a pivotal role in neuroinflammation by modulating TNF- $\alpha$ activation. J Immunol. 2014; 193:2384-2393.

30. Shen Y, He P, Zhong Z, McAllister C, Lindholm K. Distinct destructive signaling pathways of neuronal death in Alzheimer's disease. Trends Mol Med. 2006; 12:574-579.

31. Li MY, Lai FJ, Hsu LJ, Lo CP, Cheng CL, Tsai MS, Sze CI, Pugazhenthi S, Chang NS, Chen ST. Dramatic co-activation of WOX1 with CREB and NF-kB in delayed loss of small dorsal root ganglion neurons upon sciatic nerve transection in rats. PLoS One. 2009; 4:e7820.

32. Lanni C, Nardinocchi L, Puca R, Stanga S, Uberti D, Memo M, Govoni S, D’Orazi G, Racchi M. Homeodomain interacting protein kinase 2: A target for Alzheimer's beta amyloid leading to misfolded p53 and inappropriate cell survival. PLoS One. 2010; 5:e10171.

33. Stanga S, Lanni C, Govoni S, Uberti D, D’Orazi G, Racchi M: Unfolded p53 in the pathogenesis of Alzheimer's disease: is HIPK2 the link? Aging. 2010; 2:545-554.

34. Li HL, Wang HH, Liu SJ, Deng YQ, Zhang YJ, Tian Q, 
Wang XC, Chen XQ, Yang Y, Zhang JY, Wang JY, Xu $\mathrm{H}$, Liao FF, Wang JZ. Phosphorylation of tau antagonizes apoptosis by stabilizing beta-catenin, a mechanism involved in Alzheimer's neurodegeneration. Proc Natl Acad Sci USA. 2007; 104:3591-3596.

35. Aqeilan RI, Croce CM. WWOX in biological control and tumorigenesis. J Cell Physiol. 2007; 212:307-310.

36. Gomez-Ramos A, Diaz-Nido J, Smith MA, Perry G, Avila J. Effect of the lipid peroxidation product acrolein on tau phosphorylation in neural cells. J Neurosci Res. 2003; 71:863-870.

37. de PaulaVJR, Guimarães FM, Diniz BS, Forlenza OV. Neurobiological pathways to Alzheimer's disease Amyloidbeta, Tau protein or both? Dementia \& Neuropsychologia 2009; 3:188-194.

38. Goedert M, Jakes R, Qi Z, Wang JH, Cohen P. Protein phosphatase $2 \mathrm{~A}$ is the major enzyme in brain that dephosphorylates tau protein phosphorylated by prolinedirected protein kinases or cyclic AMP-dependent protein kinase. J Neurochem. 1995; 65: 2804-2807.

39. Ueberham U, Ueberham E, Bruckner MK, Seeger G, Gartner U, Gruschka H, Gebhardt R, Arendt T. Inducible neuronal expression of transgenic TGF-beta1 in vivo: dissection of short-term and long-term effects. Eur J Neurosci. 2005. 22:50-64.

40. Lee MH, Lin SR, Chang JY, Schultz L, Heath J, Hsu LJ, Kuo YM, Hong Q, Chiang MF, Gong CX, Sze CI, Chang NS. TGF-beta induces TIAF1 self-aggregation via type II receptor-independent signaling that leads to generation of amyloid beta plaques in Alzheimer's disease. Cell Death Dis. 2010; 1:e110

41. Burton T, Liang B, Dibrov A, Amara F. Transforming growth factor-beta-induced transcription of the Alzheimer beta-amyloid precursor protein gene involves interaction between the CTCF-complex and Smads. Biochem Biophys Res Commun. 2002; 295: 713-723.

42. Docagne F, Gabriel C, Lebeurrier N, Lesne S, Hommet Y, Plawinski L, Mackenzie, ET, Vivien D. Sp1 and Smad transcription factors co-operate to mediate TGF-betadependent activation of amyloid-beta precursor protein gene transcription. Biochem J. 2004; 383:393-399.

43. Hiura Y, Tabara Y, Kokubo Y, Okamura T, Miki T, Tomoike H, Iwai N. A genome-wide association study of hypertension-related phenotypes in a Japanese population. Circ J. 2010; 74:2353-2359.

44. Yang HC, Liang YJ, Chen JW, Chiang KM, Chung CM, Ho HY, Ting CT, Lin TH, Sheu SH, Tsai WC, Chen JH, Leu HB, Yin WH, Chiu TY, Chern CL, Lin SJ, Tomlinson B, Guo Y, Sham PC, Cherny SS, Lam TH, Thomas GN, Pan WH. Identification of IGF1, SLC4A4, WWOX, and SFMBT1as hypertension susceptibility genes in Han Chinese with a Genome-Wide Gene-Based Association Study. PLoS One. 2012; 7: e32907.

45. Li J, Liu J, Ren Y, Yang J, Liu P. Common chromosomal fragile site gene WWOX in metabolic disorders and tumors. Int J Biol Sci. 2014; 10:142-148.

46. Abdeen SK, Del Mare S, Hussain S, Abu-Remaileh M, Salah Z, Hagan J, Rawahneh M, Pu XA, Russell S, Stein JL, Stein GS, Lian JB, Aqeilan RI. Conditional inactivation of the mouse Wwox tumor suppressor gene recapitulates the null phenotype. J Cell Physiol. 2013; 228:1377-1382.

47. Iatan I, Choi HY, Ruel I, Prasad Linga Reddy MV, Kil H, Lee J, Odeh MA, Salah Z, Abu-Remaileh M, WeissglasVolkov D, Nikkola E, Civelek M, Awan Z, Croce CM, Aqeilan RI, Pajukanta P, Aldaz CM, Genest J. The WWOX gene modulates HDL and lipid Metabolism. Circ Cardiovasc Genet. 2014; 7:491-504.

48. Suzuki H, Katayama K, Takenaka M, Amakasu K, Saito K, Suzuki K. A spontaneous mutation of the Wwox gene and audiogenic seizures in rats with lethal dwarfism and epilepsy. Genes Brain Behav 2009; 8:650-660.

49. O'Keefe LV, Colella A, Dayan S, Chen Q, Choo A, Jacob R, Price G, Venter D, Richards RI. Drosophila orthologue of WWOX, the chromosomal fragile site FRA16D tumour suppressor gene, functions in aerobic metabolism and regulates reactive oxygen species. Hum Mol Genet. 2011; 20:497-4509.

50. Dayan S, O'Keefe LV, Choo A, Richards RI. Common chromosomal fragile site FRA16D tumor suppressor WWOX gene expression and metabolic reprograming in cells. Genes Chromosomes Cancer. 2013; 52:823-831.

51. Chang NS, Schultz L, Hsu LJ, Lewis J, Su M, Sze CI. 17beta-Estradiol upregulates and activates WOX1/ WWOXv1 and WOX2/WWOXv2 in vitro: potential role in cancerous progression of breast and prostate to a premetastatic state in vivo. Oncogene. 2005; 24:714-723.

52. Su WP, Chen SH, Chen SJ, Chou PY, Huang CC, Chang NS. WW Domain-containing oxidoreductase is a potential receptor for sex steroid hormones. In "Sex Hormones" edited by Dubey Raghvendra, InTech - Open Access Publisher, 2012; pp. 333-351.

53. Hasty P, Christy BA. p53 as an intervention target for cancer and aging. Pathobiol Aging Age Relat Dis. 2013;3. doi: 10.3402/pba.v3i0.22702.

54. Vigneron A, Vousden KH. p53, ROS and senescence in the control of aging. Review. Aging. 2010; 8:471-474. 